\title{
Nonlinear conductance of quantum point contacts
}

\author{
L. P. Kouwenhoven, B. J. van Wees, and C. J. P. M. Harmans \\ Department of Applied Physics, Delft University of Technology, P. O. Box 5046, 2600 GA Delft, The Netherlands \\ J. G. Williamson, H. van Houten, and C. W. J. Beenakker \\ Philips Research Laboratories, 5600 JA Eindhoven, The Netherlands \\ C. T. Foxon and J. J. Harris \\ Philips Research Laboratories, Redhill, Surrey RHI 5HA, United Kingdom
}

(Received 12 December 1988)

\begin{abstract}
The conductance of ballistic quantum constrictions in a two-dimensional electron gas has been studied experimentally as a function of the applied voltage. Large nonlinearities are found in the current-voltage characteristics. We give a simple model, which explains the main features of the nonlinear conductance. Breakdown of the conductance quantization occurs when the number of occupied one-dimensional subbands becomes unequal for the two velocity directions. A critical voltage is found for the breakdown, which is equal to the subband separation at the Fermi level.
\end{abstract}

The quantization of the conductance of a constriction in a two-dimensional electron gas (2D EG) was recently discovered in the experiments of van Wees et al. ${ }^{1}$ and Wharam et al. ${ }^{2}$ They defined a ballistic constriction in the 2D EG of a high-mobility GaAs- $\mathrm{Al}_{x} \mathrm{Ga}_{-x}$ As heterostructure by means of a metallic split gate. Application of a negative voltage $V_{g}$ on the gate forms the constriction in the 2D EG by electrostatic depletion. The two-terminal conductance $G$, measured at zero magnetic field between the two wide regions of $2 \mathrm{D}$ EG on each side of the constriction, was shown to change stepwise in units of $2 e^{2} / h$ on varying $V_{g}$. The quantization of $G$ can be explained from the formation of one-dimensional (1D) subbands in the constriction due to the lateral confinement. Then $G$ is given by the Landauer-type formula ${ }^{3} G=N_{c} 2 e^{2} / h$, with $N_{c}$ the number of occupied 1D subbands. A detailed analysis has shown that a variation of $V_{g}$ changes the width as well as the electron density of the constriction. ${ }^{4}$ Both mechanisms move the Fermi energy $E_{F}$ in the channel through the 1D subbands and whenever it passes a subband bottom $G$ changes by the quantized amount of $2 e^{2} / h$.

So far this new conductance quantization has been studied in the linear ballistic transport regime. Here we report on the nonlinear conductance of quantum point contacts. Deviations from quantization are expected to occur when $\mathrm{eV}$ becomes comparable to the subband separation (with $V$ the applied voltage over the constriction). We have studied the nonlinear transport by measuring a set of current-voltage $(I-V)$ characteristics using $V_{g}$ as a parameter. The main features of the $I-V$ characteristics can be accounted for by a simple qualitative model, which is based on ballistic electron transport over a potential barrier in the constriction. Related models have been used in the field of hot electron transport in layered semiconductor structures, ${ }^{5}$ and to explain the breakdown of the quantum Hall effect. ${ }^{6-10}$

The measurements have been performed on a device which is similar to that in Ref. 1 (see inset Fig. 1). The
2D EG of the GaAs- $\mathrm{Al}_{x} \mathrm{Ga}_{1-x} \mathrm{As}$ heterostructure has a transport mean free path of $8.5 \mu \mathrm{m}$ and an electron density of $3.6 \times 10^{15} / \mathrm{m}^{2}$ resulting in a Fermi wavelength of 42 $\mathrm{nm}$. At a gate voltage $V_{g}=-0.6 \mathrm{~V}$ the constriction is just formed in the 2D EG and has its maximum width, which is approximately equal to the lithographic width of the opening in the gate $(250 \mathrm{~nm})$. Lowering $V_{g}$ reduces the width and at $V_{g}=-2.2 \mathrm{~V}$ the constriction is fully pinched off. The experiments were done at $0.6 \mathrm{~K}$ with dc current biasing. The voltage $V$ across the constriction is defined as the voltage of the upper contact in the inset of Fig. 1 minus the voltage of the lower contact. The measured voltage is corrected for a background resistance originating from the two wide $2 \mathrm{D}$ EG regions and from the resistance of the Ohmic contacts. ${ }^{11}$

In Fig. 1 the $I-V$ characteristics are shown for several values of the gate voltage $V_{g}$, for which the constriction is pinched off in equilibrium. For low $V$ the current through the constriction is zero. At a certain critical voltage $V_{c}$ there is a stepwise increase of the differential conductance

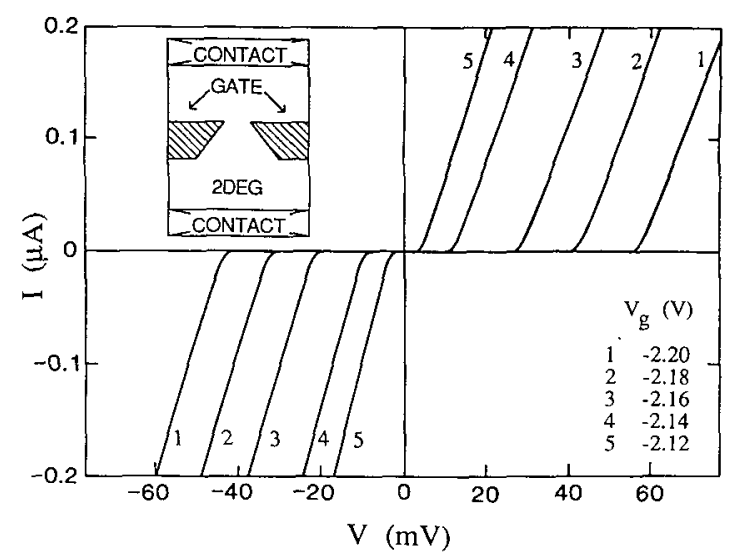

FIG. 1. $I-V$ characteristics at different values of gate voltage $V_{g}$ for which the constriction is pinched off for small voltage $V$. The inset shows the sample layout. 
$g=\partial I / \partial V$ from zero to a constant value $\left[\approx(80 \mathrm{k} \Omega)^{-1}\right]$, which is found to be nearly independent of $V_{g}$. The critical voltage $V_{c}$, however, increases strongly with decreasing $V_{g}$. Note that the $I-V$ characteristics are not antisymmetric. The asymmetry is considerably influenced by the choice of zero reference of $V_{g}$, which in the experiment has been the lower contact in the sample layout of Fig. 1. Changing the zero reference to the upper contact results in a different gate voltage $V_{g}+V$, which gives for $V<0$ a lower gate voltage. However, this change in zero reference does not account for the asymmetry in the curves of Fig. 1. This might be due to an intrinsic asymmetry in the electrostatic potential defining the constriction. In a second device of identical design the change of zero reference completely accounted for the asymmetry.

In Fig. 2 the $I-V$ characteristics are shown for a range of $V_{g}$, for which the constriction is already conducting at a small applied voltage $V$. For comparison we display $G$ at small $V$ as a function of $V_{g}$ for the lowest two quantized plateaus in the inset of Fig. 2(a). As can be seen in the inset, $V_{g}$ ranges from near pinch off $\left(V_{g}=-2.10 \mathrm{~V}\right)$ to the onset of the second plateau $\left(V_{g}=-2.00 \mathrm{~V}\right)$. In Fig. 2(a)
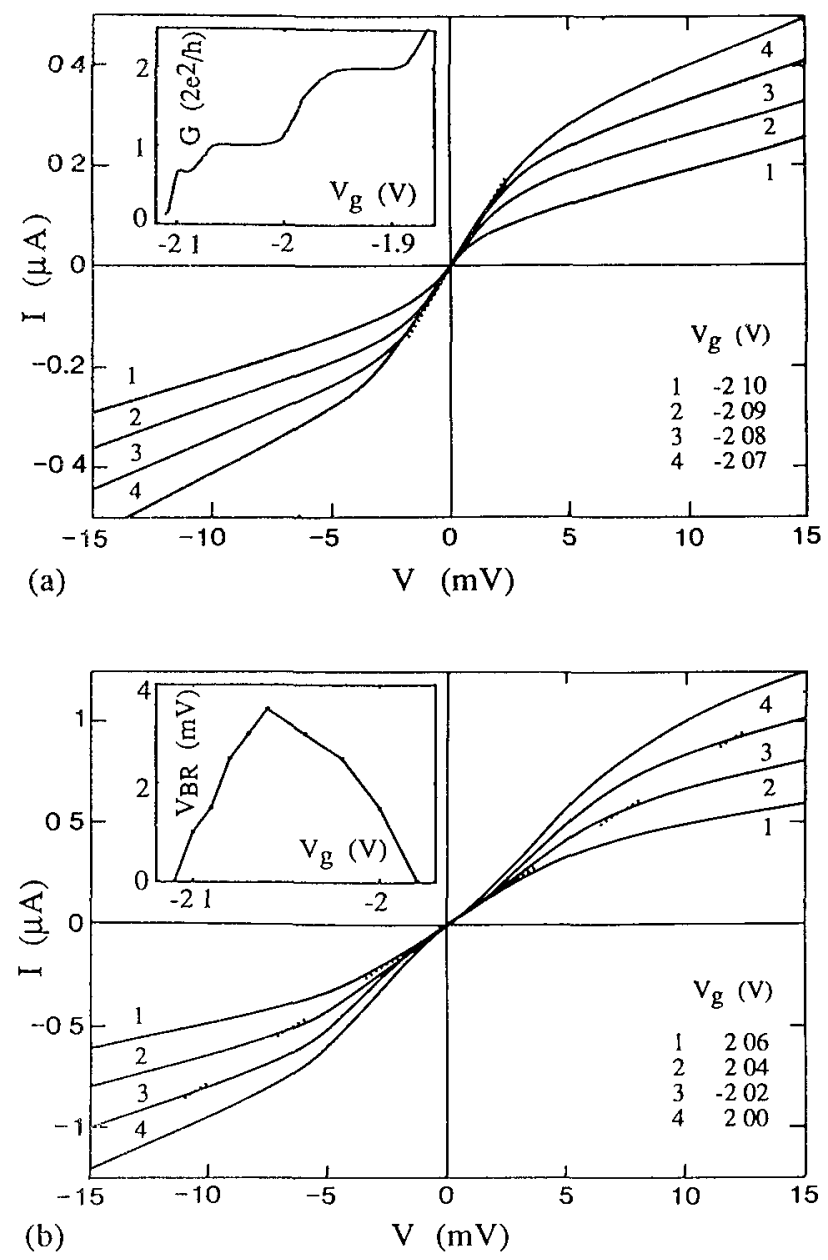

FIG $2 I-V$ characteristics at different values of $V_{g}$ for which the conductance $G$ is quantized at $2 e^{2} / h$ for small $V$, indicated by the dotted line. The inset of (a) shows $G$ as function of $V_{g}$ in equilibrium and the inset of (b) the breakdown voltage $V_{\mathrm{BR}}$ as a function of $V_{g}$ the curves are displayed for gate voltages corresponding to the lower part of the first plateau. For small $V$ they follow the dotted line, which indicates the quantized value $2 e^{2} / h$ of the first plateau. At a certain voltage $V_{\mathrm{BR}}$, the quantization breaks down and $g$ decreases from $2 e^{2} / h[\approx(13$ $\left.\mathrm{k} \Omega)^{-1}\right]$ to a lower value $\left[\approx(60 \mathrm{k} \Omega)^{-1}\right]$. In Fig. 2(b) the $I-V$ characteristics are shown for gate voltages corresponding to the upper part of the first plateau. Again the curves follow the dotted line of quantization for small $V$ and deviate from it above a breakdown voltage. However in contrast to Fig. 2(a) the deviation from quantization is now to a larger value for $g\left[\approx(8.7 \mathrm{k} \Omega)^{-1}\right]$. A further increase of $V$ reduces $g$ to a value much lower than $2 e^{2} / h$. Note that the relative effect of $V$ on the gate voltage and hereby the asymmetry is much less in Fig. 2 as compared to Fig. 1. We thus see that increasing $V$ results in the breakdown of the conductance quantization, as manifested by either an increase or a decrease in $g$. As can be seen from Fig. 2, the breakdown voltage $V_{\mathrm{BR}}$ (the voltage where $g$ deviates more than $10 \%$ from the quantized value) increases as $V_{g}$ approaches the center of the first plateau. To illustrate this we have plotted $V_{\mathrm{BR}}$ as a function of $V_{g}$ in the inset of Fig. 2(b), which shows a triangular shape with a maximum value of $3.5 \mathrm{meV}$ at $V_{g}$ $=-2.06 \mathrm{~V}$.

To understand the main features in the $I-V$ characteristics we propose a simple model. Apart from the lateral confinement, the gate voltage $V_{g}$ also gives rise to an electrostatic potential in the constriction, ${ }^{4,12}$ which results in a reduced electron density. For simplicity we neglect the voltage $V$ dependence of $V_{g}$. Due to the lateral confinement $1 \mathrm{D}$ subbands are formed. On entering the constriction the bottom of the $n$th subband rises relative to the bulk 2D EG, as a combined result of the increased lateral confinement and the electrostatic barrier. The number of occupied states is lowest at the maximum of the potential barrier, where the $n$th subband bottom has an energy $E_{n}$ constituting a "bottleneck" for the current. Extrapolating an approach valid in the linear transport regime, ${ }^{3,12}$ we calculate the net current $I_{n}$ through the constriction carried by the $n$th subband by considering the occupation of the right- and left-moving states at the bottleneck $E_{n}$. The right-moving states are filled from $E_{n}$ up to $\mu_{1}$, the electrochemical potential at the left of the constriction (provided that $\mu_{1}>E_{n}$ ). Analogously, provided that $\mu_{2}>E_{n}$, the left-moving states are filled from $E_{n}$ up to $\mu_{2}$, the electrochemical potential at the right. We assume that the electrons with energy $\mu>E_{n}$ are fully transmitted through the constriction. A difference in occupation between the right- and left-moving states is determined by the applied voltage $V$, with $e V=\mu_{1}-\mu_{2}$ (assuming a vanishing electric field outside the constriction), resulting in a net current. For $\mu_{1}>\mu_{2}$ the $n$th subband carries a net current, which according to the well-known cancellation of group velocity with density of states in one dimension is given by

$$
I_{n}=(2 e / h)\left[\mu_{1}-\max \left(\mu_{2}, E_{n}\right)\right]
$$

provided $\mu_{1}>\max \left(\mu_{2}, E_{n}\right)$, and $I_{n}=0$ otherwise. On increasing $V$ the population of the right-moving states increases to $\mu_{1}=E_{F}+m e V$ and of the left-moving states de- 
creases to $\mu_{2}=E_{F}-(1-m) e V$. Here $m$ is a phenomenological parameter between 0 and 1 , describing the fraction of $V$, which drops on the left of the bottleneck. Concurrently the fraction $(1-m)$ of $V$ drops on the right. At a certain voltage $\mu_{1}$ or $\mu_{2}$ crosses the subband bottom $E_{n}$, in this way changing the contribution $\partial I_{n} / \partial V$ from the $n$th subband to the differential conductance $g$. We find for $E_{F}<E_{n}$

$$
\frac{\partial I_{n}}{\partial V}=\left\{\begin{array}{l}
0, \text { if }|V|<V_{c}=-\left(E_{F}-E_{n}\right) / m e, \\
m 2 e^{2} / h, \text { if }|V|>V_{c},
\end{array}\right.
$$

while if $E_{F}>E_{n}$

$\frac{\partial I_{n}}{\partial V}=\left\{\begin{array}{l}2 e^{2} / h, \text { if }|V|<V_{c}^{\prime}=\left(E_{F}-E_{n}\right) /(1-m) e, \\ m 2 e^{2} / h, \text { if }|V|>V_{c}^{\prime} .\end{array}\right.$

Equation (2) applies to a subband which in equilibrium is not occupied at the bottleneck of the constriction $\left(E_{F}<E_{n}\right)$. The differential conductance from this subband increases beyond a critical voltage $V_{c}$ to a value which is smaller than the quantized value. Equation (3) applies to a subband which is occupied in equilibrium $\left(E_{F}>E_{n}\right)$. Beyond a critical voltage $V_{c}^{\prime}$ the differential conductance due to this subband decreases from its normal quantized value of $2 e^{2} / h$. Although the expressions for the critical voltages depend on the parameter $m$, these conclusions are general and model independent.

To illustrate the consequences of Eqs. (2) and (3) on increasing the voltage we have schematically shown in Fig. 3 the energy of the two lowest subbands at the bottleneck as a function of longitudinal wave vector $k_{y}$. Note that positive $k_{y}$ corresponds to a positive velocity. In equilibrium $(V=0)$ the subbands are occupied up to the Fermi energy $E_{F}$. A voltage $V$ across the constriction gives a difference $\mu_{1}-\mu_{2}=e V$ in occupation between the two ve-

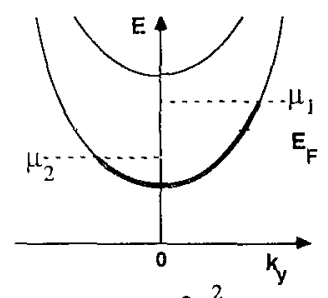

(a) $\quad g=\frac{2 e^{2}}{h}$

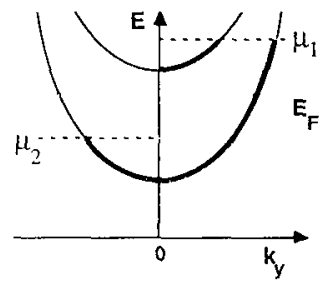

(c) $g=(1+m) \frac{2 \mathrm{e}^{2}}{\mathrm{~h}}$ (b)

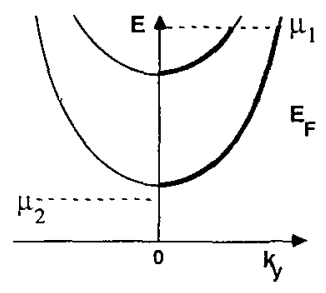

(d) $\quad \mathrm{g}=2 \mathrm{~m} \frac{2 \mathrm{e}^{2}}{\mathrm{~h}}$

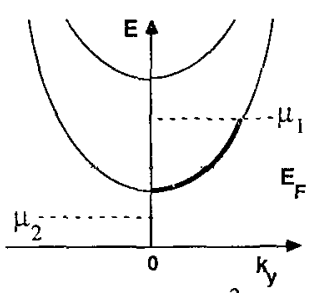

FIG. 3. Subband occupation at the bottleneck, where the conductance is determined. Four situations are illustrated for different $V$ across the constriction (with $e V=\mu_{1}-\mu_{2}$ ), and for different positions of $E_{F}$. locity directions [Fig. 3(a)], resulting in a net current. As long as the number of occupied subbands is the same for both velocity directions the conductance is quantized. However, at larger applied voltages, $\mu_{2}$ can fall below the bottom of a subband. Here $g$ reduces from $2 e^{2} / h$ to a fraction $m 2 e^{2} / h$, as shown in Fig. 3(b) (where $E_{F}$ is near the bottom of the lowest subband) and as observed experimentally in Fig. 2(a). The subband occupation of Fig. 3(b) can also be reached from the situation $E_{F}<E_{1}$, where there are no occupied states in equilibrium. For low voltages $g=0$ as in Fig. 1, but at a critical voltage $\mu_{1}$ crosses $E_{1}$ and $g$ increases to $m 2 e^{2} / h$ according to Eq. (2). We emphasize that this explanation for the onset of conductance holds that $\mu_{1}$ is lifted above the barrier in the constriction. The constant $g$ above the critical voltage excludes tunneling through the barrier, which would lead to an exponential dependence of $g$ on $V .{ }^{13}$ Figures 3(c) and $3(\mathrm{~d})$ correspond to the situation where $E_{F}$ is close to the bottom of the second subband, as in the experimental Fig. 2(b). On increasing $V$ first the second subband starts to be populated [Fig. 3(c)] leading to an increase of $g$ to $(1+m) 2 e^{2} / h$. A further increase of $V$ causes $\mu_{2}$ to fall below the bottom of the first subband [Fig. 3(d)], which then reduces $g$ to a fraction $2 m 2 e^{2} / h$. This explains qualitatively the increasing and then decreasing slope in Fig. 2(b). We note that the situation of Fig. 3(d) can also be reached directly from Fig. 3(a), which is actually happening at $V_{g}=-2.06 \mathrm{~V}$ in Fig. 2(b). The model presented here in terms of a single phenomenological parameter $m$ does give qualitative insight, but it is not a realistic description of the complex interdependence of the electrostatic potential on $V$ and $V_{g}$. This is demonstrated by the fact that no universal value for $m$ is found. If both velocity directions are occupied the experiment yields $m \approx 0.5$. The maximum of the breakdown voltage $V_{\mathrm{BR}}$ at $V_{g}=-2.06 \mathrm{~V}$ (for which $E_{F}$ is approximately in the middle of the first and second subband bottom as can be seen from the insets of Fig. 2), also indicates $m \approx 0.5$. However, if one velocity direction is fully depopulated, $m$ has an experimental value of $\approx 0.2$. It would be of interest to develop a more quantitative theory for our observations.

It follows from Eqs. (2) and (3) that the maximum value of the breakdown voltage $V_{\mathrm{BR}}$ is equal to the subband separation at the Fermi level. This is independent of the parameter $m$ and provides a fundamental limit for the conductance quantization. From the inset of Fig. 2(b) we thus find a subband separation of $3.5 \mathrm{meV}$, which is consistent with the value obtained from an analysis of magnetic depopulation. ${ }^{4,14}$ As we have discussed, the breakdown of the conductance quantization occurs whenever the number of occupied subbands differs for the two velocity directions. We emphasize that this mechanism does not involve any inelastic process or intersubband scattering. The triangular dependence of the breakdown voltage [see inset Fig. 2(b)] on the gate voltage is reminiscent of experiments on the breakdown of the quantum Hall effect, where a similar dependence of the breakdown Hall voltage on the magnetic field was found. ${ }^{6-9}$ A mechanism for breakdown of the quantum Hall effect also including only elastic processes has been proposed in Ref. 10 .

In this paper we have presented $I-V$ characteristics at 
fixed $V_{g}$. Glazman and Khaetskii ${ }^{15}$ have recently predicted that the differential conductance as a function of gate voltage at a fixed finite $V$ should exhibit additional plateaus in between the plateaus at multiples of $2 e^{2} / h$. We have found some evidence for these additional plateaus [which follow also from Eq. (2)], but these are not well resolved in our device.

In conclusion, we have reported the first experimental study on the nonlinear behavior of quantum ballistic point contacts. We have given a simple model explaining the main features in the nonlinear conductance. The measured $I-V$ characteristics reveal the occupation of the $1 \mathrm{D}$ subbands formed in the constriction, for the individual velocity directions. Breakdown of the quantization occurs when the number of occupied subbands becomes different for the two directions. A critical voltage equal to the subband separation at the Fermi level is derived for the complete breakdown of the two-terminal conductance quantization.

We thank M. E. I. Broekaart, C. E. Timmering, and L. W. Lander for technical support and L. J. Geerligs and E. M. M. Willems for assistance with the experiments. We thank J. Romijn and the Delft Centre for Submicron Technology (CST) for the facilities offered and the "Stichting voor Fundamenteel Onderzoek der Materie" (FOM) for financial support. One of us (L.P.K.) gratefully acknowledges the financial support of the CST.

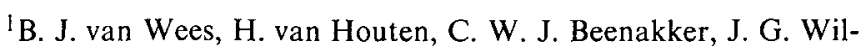
liamson, L. P. Kouwenhoven, D. van der Marel, and C. T. Foxon, Phys. Rev. Lett. 60, 848 (1988).

${ }^{2}$ D. A. Wharam, T. J. Thornton, R. Newbury, M. Pepper, H. Ahmed, J. E. F. Frost, D. G. Hasko, D. C. Peacock, D. A. Ritchie, and G. A. C. Jones, J. Phys. C 21, L209 (1988).

${ }^{3}$ R. Landauer, Z. Phys. B 68, 217 (1987).

${ }^{4}$ B. J. van Wees, L. P. Kouwenhoven, H. van Houten, C. W. J. Beenakker, J. E. Mooij, C. T. Foxon, and J. J. Harris, Phys. Rev. B 38, 3625 (1988).

${ }^{5}$ J. R. Hayes, A. F. J. Levi, and W. Wiegman, Phys. Rev. Lett. 54, 1570 (1985); M. Heiblum, M. I. Nathan, D. C. Thomas, and C. M. Knoedler, ibid. 55, 2200 (1985).

${ }^{6} \mathrm{G}$. Ebert, K. von Klitzing, K. Ploog, and G. Weimann, J. Phys. C 16, I5441 (1983).

${ }^{7}$ J. R. Kirtley, Z. Schlesinger, T. N. Theis, F. P. Milliken, S. L. Wright, and L. F. Palmateer, Phys. Rev. B 34, 5414 (1986).

${ }^{8}$ L. Bliek, E. Braun, G. Hein, V. Kose, J. Niemeyer, G. Weiman, and W. Schlapp, Semicond. Sci. Technol. 1, 110 (1986).

${ }^{9}$ P. G. N. de Vegvar, A. M. Chang, G. Timp, P. M. Mankiewich, J. E. Cunningham, R. Behringer, and R. E. Howard, Phys.
Rev. B 36, 9366 (1987).

${ }^{10}$ J. K. Jain and S. A. Kivelson, Phys. Rev. B 37, 4276 (1988).

${ }^{11}$ The resistance of the Ohmic contacts showed to be slightly nonlinear. It is measured independently at $V_{g}=0 \mathrm{~V}$, where it varied within $2.9 \pm 0.4 \mathrm{k} \Omega$ over the current range and is subtracted from the measured data. The formation of the constriction at $V_{g}=-0.6 \mathrm{~V}$ gives rise to a series resistance $R_{2 \mathrm{DEG}}$ from the two wide $2 \mathrm{D}$ EG regions, due to the change in geometry. $R_{2 \mathrm{DEG}}(=980 \Omega$ ) has been obtained from the difference of the measured and quantized value of the first plateau and is taken constant for all $I-V$ curves.

${ }^{12}$ B. J. van Wees, H. van Houten, C. W. J. Beenakker, L. P. Kouwenhoven, J. G. Williamson, J. E. Mooij, C. T. Foxon, and J. J. Harris, in Proceedings of the Nineteenth International Conference on the Physics of Semiconductors, Warsaw, Poland, 1988 (unpublished).

${ }^{13}$ A. Palevski, M. Heiblum, C. P. Umbach, C. M. Knoedler, A. N. Broers, and R. H. Koch (unpublished).

${ }^{14}$ B. J. van Wees et al. (unpublished).

${ }^{15}$ L. I. Glazman and A. V. Khaetskii (unpublished). 